\title{
Supporting Information Synthesis and Laser Ablation Mass Spectrometry of Nitrogen-doped Carbon Materials
}

Brett A. Williams, Marissa A. Dobulis, Yaroslav Losovyj, Ulrike Werner-Zwanziger, A. R. Siedle, ${ }^{*}$ and Caroline Chick Jarrold*

Department of Chemistry, Indiana University, 800 E. Kirkwood Ave, Bloomington, IN 47405

*Authors to whom correspondence should be addressed. E-mail: cjarrold@indiana.edu; arsiedle@indiana.edu 


\section{Table of Contents}

S3 Bulk material characterization: XPS Studies.

S15

Bulk material characterization: Laser ablation/mass spectrometric analysis. S16 Bulk material characterization: References

Table S1: XPS atomic \% contents

Figure S1: Survey XPS spectra

Figure S2: Valence band XPS spectra

Tables S2 and S3: Deconvolution tables for XPS spectrum of Sample 1

Tables S4 and S5: Deconvolution tables for XPS spectrum of Sample 2

Tables S6 and S7: Deconvolution tables for XPS spectrum of $\mathrm{C}_{3} \mathrm{~N}_{4}$

Figure S3: Mass spectrum of $\mathbf{1}$ with odd-mass copper carbide clusters

Figure S4: Mass spectra from Fig. 6(d) shown over full $\mathrm{m} / \mathrm{z}$ range

Laser desorption/ionization mass spectral analysis.
Figure S5: Mass spectrum for anions generated from matrix-free LDI of $\mathrm{C}_{3} \mathrm{~N}_{4}$

Figure S6: Mass spectrum for cations generated from matrix-free LDI of $\mathrm{C}_{3} \mathrm{~N}_{4}$

Table S8: Major mass patterns for peaks in Figure S5

Table S9: Major mass patterns for peaks in Figure S6

Table S10: List of all masses in Figure S5 along with likely assignments

Table S11: List of all masses in Figure S6 along with likely assignments

Figure S7: Energies and structures of $\mathrm{C}_{4} \mathrm{~N}_{2} \mathrm{H}_{6}$ anion and neutral species

Figure S8: Energies and structures of $\mathrm{C}_{4} \mathrm{~N}_{2}$ anion and neutral species

Table S12: Timing delays between the various triggers for laser ablation PXRD data collection procedure.

Figure S9: PXRD spectrum for 1

Figure S10: PXRD spectrum for 2 


\section{Bulk material characterization}

XPS Studies. The XPS experiments were carried out using PHI VersaProbe II instrument equipped with a focused monochromatic $\mathrm{Al} \mathrm{K \alpha}$ source. Instrument base pressure was ca. $8 \times 10^{-10}$ Torr. The X-ray power of $46 \mathrm{~W}$ at $15 \mathrm{kV}$ was used for XPS spectra acquisition mode with $200 \mu \mathrm{m}$ beam size at the normal X-ray incidence and take off angles of $45^{\circ}$. Calibration of the instrument analyzer work function was set to give a binding energy (BE) of $84.0 \mathrm{eV}$ for Au $4 f_{7 / 2}$ line for metallic gold. The spectrometer dispersion was adjusted to give BE's of $284.8 \mathrm{eV}, 932.7 \mathrm{eV}$ and of $368.3 \mathrm{eV}$ for the $\mathrm{C} 1 s$ line of adventitious (aliphatic) carbon presented on the non-sputtered samples, $\mathrm{Cu} 2 p_{3 / 2}$ and $\mathrm{Ag} 3 d_{5 / 2}$ photoemission lines, respectively. The PHI dual charge neutralization system was used on all samples. The high resolution $\mathrm{N} 1 \mathrm{~s}, \mathrm{O} 1 \mathrm{~s}, \mathrm{C} 1 \mathrm{~s}$ and $\mathrm{F} 1 \mathrm{~s}$ spectra were taken with a minimum of 10-60 s scans using $0.1 \mathrm{eV}$ steps and $23.5 \mathrm{eV}$ pass energy. At the ultimate PHI Versa Probe II instrumental resolution, the room temperature spread (at 14/86\%) of the metallic silver Fermi edge was less than $120 \mathrm{meV}$. All XPS spectra were recorded using PHI software SmartSoft -XPS v2.6.3.4 and processed using PHI MultiPack v9.3.0.3 and/or CasaXPS v.2.3.14 using Shirley background. The relative sensitivity factors from MultiPack library were used to determine atomic percentages. Peaks were fitted using GL line shapes a combination of Gaussians and Lorentzians. A given sample was examined in at least 3 different spots on the mounted specimen to assure that consistent, reproducible results were obtained. All HR spectra were calibrated in respect to $s p^{2} \mathrm{C} 1 \mathrm{~s}$ of HOPG. 
Laser ablation/mass spectrometric analysis. The details of the experimental apparatus used in this study have been described elsewhere. ${ }^{1-3}$ Anions are generated using a laser ablation/pulsed molecular beam valve source coupled to a 25-mm long, 3-mm diameter reaction channel. Approximately 4-10 mJ/pulse of the second harmonic output $(532 \mathrm{~nm}, 2.33 \mathrm{eV})$ of a Nd:YAG laser, operated at a repetition rate of $30 \mathrm{~Hz}$, was focused onto the surface of a target prepared by hydraulically pressing samples (detailed below). The target was disk shaped and rotated in the source to provide fresh sample for each ablation event. The resulting plasma was swept through the channel by a pulse of ultrahigh purity (UHP) He carrier gas (30 psig stagnation pressure) and expanded into a vacuum chamber. The expansion was skimmed and the anions were selectively accelerated on axis into a Bakker-style beam-modulated time-of-flight (BMTOF) mass spectrometer. ${ }^{4}$ The anions then traveled 1.2 meters before being detected by a dual microchannel plate (MCP) detector assembly.

In order to compare our samples to a fully-stoichiometric C-N species, graphitic carbon nitride $\left(\mathrm{C}_{3} \mathrm{~N}_{4}\right)$ was analyzed using matrix-free laser desorption/ionization (LDI) time-of-flight mass spectrometry. ${ }^{5-6} \mathrm{~A}$ small amount of the sample was transferred to the MALDI plate and a pulse of a Nd:YAG laser (355 nm, $3.49 \mathrm{eV}$ ) operated at $50 \mathrm{~Hz}$ irradiated the sample; the laser power was adjusted to a threshold level, i.e. just high enough to generate ions. This process was performed for both positive and negative ions. The TOF mass spectrometer was calibrated using cluster ions from $\alpha$-cyano-4-hydroxycinnamic acid (CCA), a well-known small-molecule matrix. ${ }^{6-7}$ 


\section{References}

1. Williams, B. A.; Siedle, A. R.; Jarrold, C. C. Evidence of $\mathrm{CF}_{2}$ Loss from Fluorine-Rich Cluster Anions Generated from Laser Ablation of Graphite Fluoride. J. Phys. Chem. A 2018, 122, 9894-9900.

2. Moravec, V. D.; Jarrold, C. C. Study of the Low-Lying States of $\mathrm{NiO}^{-}$and NiO Using Anion Photoelectron Spectroscopy. J. Chem. Phys. 1998, 108, 1804-1810.

3. Waller, S. E.; Mann, J. E.; Jarrold, C. C. Asymmetric Partitioning of Metals among Cluster Anions and Cations Generated Via Laser Ablation of Mixed Aluminum/Group 6 Transition Metal Targets. J. Phys. Chem. A 2013, 117, 1765-1772.

4. Bakker, J. M. B. A Beam-Modulated Time-of-Flight Mass Spectrometer. II. Experimental Work. J. Phys. E: Sci. Instrum 1974, 7 , 364.

5. Mandal, A.; Singha, M.; Addy, P. S.; Basak, A. Laser Desorption Ionization Mass Spectrometry: Recent Progress in Matrix-Free and Label-Assisted Techniques. Mass Spectrom. Rev. 2019, 38, 3-21.

6. Krutchinsky, A. N.; Chait, B. T. On the Nature of the Chemical Noise in MALDI Mass Spectra. J. Am. Soc. Mass Spectrom. 2002, $13,129-134$.

7. Harris, W. A.; Janecki, D. J.; Reilly, J. P. Use of Matrix Clusters and Trypsin Autolysis Fragments as Mass Calibrants in MatrixAssisted Laser Desorption/Ionization Time-of-Flight Mass Spectrometry. Rapid Commun. Mass Spectrom. 2002, 16, 1714-1722. 
Table S1. XPS atomic \% contents

\begin{tabular}{|c|c|c|c|c|}
\hline At\% & C1s & N1s & O1s & F1s \\
\hline $\mathbf{1}$ & 81.1 & 11.6 & 6.8 & 0.5 \\
\hline $\mathbf{2}$ & 59.2 & 30.7 & 8.1 & 1.9 \\
\hline
\end{tabular}


Figure S1. Survey XPS spectra

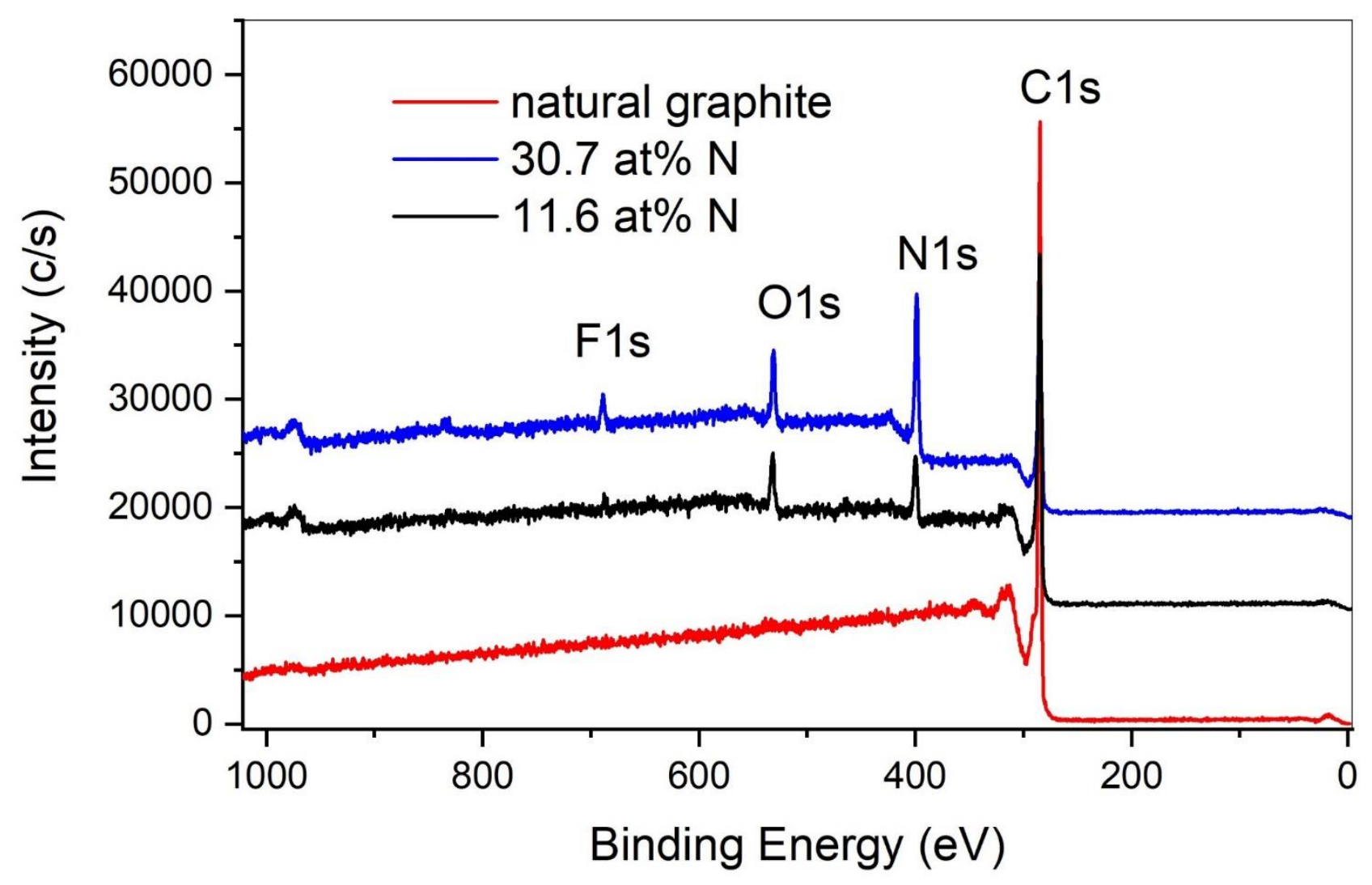


Figure S2. Valence band XPS spectra

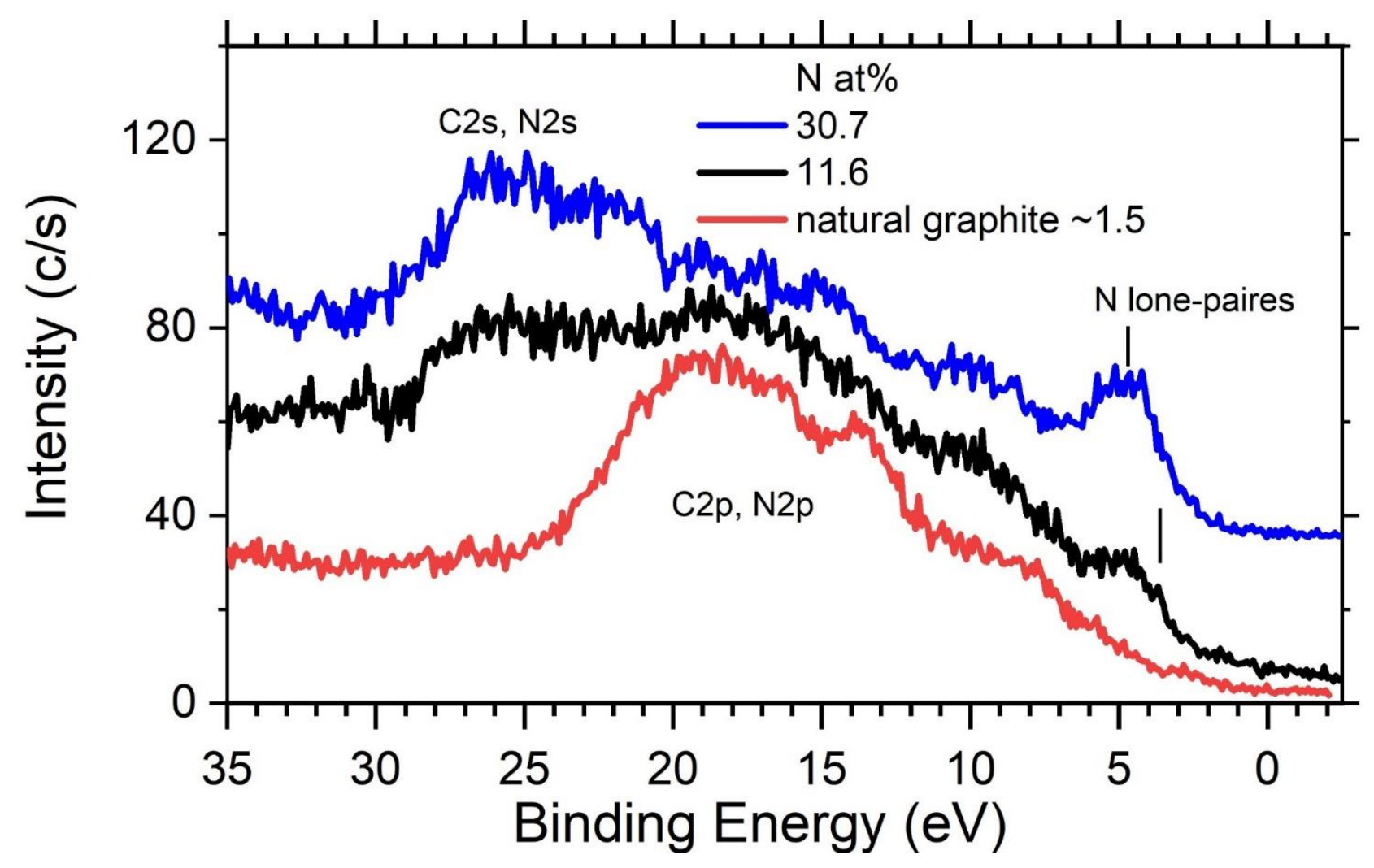


Table S2 and Table S3. Deconvolution tables for XPS spectrum of Sample 1

C1s

\begin{tabular}{|c|c|c|c|c|c|c|}
\hline Band & Pos & PosSep & FWHM & \%Gauss & \%Area & ChiSquared \\
\hline 1 & 284.43 & 0.00 & 0.90 & 90 & 30.87 & 6.59 \\
\hline 2 & 285.13 & 0.70 & 1.66 & 100 & 27.48 & \\
\hline 3 & 285.58 & 1.15 & 2.95 & 90 & 8.95 & \\
\hline 4 & 286.17 & 1.74 & 1.86 & 94 & 12.80 & \\
\hline 5 & 287.18 & 2.75 & 2.51 & 100 & 5.56 & \\
\hline 6 & 288.60 & 4.17 & 2.77 & 91 & 8.19 & \\
\hline 7 & 291.13 & 6.70 & 4.52 & 97 & 6.14 & \\
\hline
\end{tabular}

N1s

\begin{tabular}{|c|c|c|c|c|c|c|}
\hline Band & Pos & PosSep & FWHM & \%Gauss & \%Area & ChiSquared \\
\hline 1 & 398.90 & 0.00 & 2.06 & 87 & 41.20 & 1.79 \\
\hline 2 & 400.45 & 1.55 & 2.36 & 90 & 53.56 & \\
\hline 3 & 403.90 & 5.00 & 2.91 & 90 & 5.24 & \\
\hline
\end{tabular}


Table S4 and Table S5. Deconvolution tables for XPS spectrum of Sample 2

C1s

\begin{tabular}{|c|c|c|c|c|c|c|}
\hline Band & Pos & PosSep & FWHM & \%Gauss & \%Area & ChiSquared \\
\hline 1 & 284.40 & 0.00 & 1.10 & 100 & 18.03 & 2.45 \\
\hline 2 & 285.62 & 1.22 & 1.75 & 100 & 10.03 & \\
\hline 3 & 285.75 & 1.35 & 1.80 & 87 & 35.10 & \\
\hline 4 & 286.47 & 2.07 & 1.65 & 100 & 8.36 & \\
\hline 5 & 287.00 & 2.60 & 2.03 & 100 & 14.42 & \\
\hline 6 & 288.60 & 4.20 & 2.31 & 88 & 9.55 & \\
\hline 7 & 291.10 & 6.70 & 3.76 & 100 & 4.51 & \\
\hline
\end{tabular}

N1s

\begin{tabular}{|c|c|c|c|c|c|c|}
\hline Band & Pos & PosSep & FWHM & \%Gauss & \%Area & ChiSquared \\
\hline 1 & 398.63 & 0.00 & 1.81 & 90 & 28.24 & 1.98 \\
\hline 2 & 400.06 & 1.43 & 2.57 & 82 & 68.53 & \\
\hline 3 & 403.13 & 4.50 & 2.90 & 70 & 3.22 & \\
\hline
\end{tabular}


Table S6 and Table S7. Deconvolution tables for XPS spectrum of $\mathrm{C}_{3} \mathrm{~N}_{4}$

C1s

\begin{tabular}{|c|c|c|c|c|c|c|}
\hline Band & Pos & PosSep & FWHM & \%Gauss & \%Area & ChiSquared \\
\hline 1 & 284.39 & 0.00 & 0.90 & 90 & 5.35 & 1.97 \\
\hline 2 & 285.06 & 0.67 & 1.10 & 90 & 14.44 & \\
\hline 3 & 286.13 & 1.74 & 1.89 & 90 & 10.16 & \\
\hline 4 & 288.15 & 3.76 & 1.72 & 99 & 57.28 & \\
\hline 5 & 288.59 & 4.20 & 1.70 & 90 & 9.63 & \\
\hline 6 & 292.82 & 8.44 & 1.33 & 90 & 3.14 & \\
\hline
\end{tabular}

N1s

\begin{tabular}{|c|c|c|c|c|c|c|}
\hline Band & Pos & PosSep & FWHM & \%Gauss & \%Area & ChiSquared \\
\hline 1 & 398.72 & 0.00 & 1.69 & 90 & 69.43 & 1.50 \\
\hline 2 & 400.42 & 1.70 & 1.59 & 90 & 25.71 & \\
\hline 3 & 404.02 & 5.30 & 2.14 & 90 & 4.85 & \\
\hline
\end{tabular}


Figure S3. Mass spectrum of Sample 1 collected under conditions which produce odd-mass copper carbide clusters. The ion signal in the red box is shown on an expanded scale (inset) to show and identify these clusters. The ion at $\mathrm{m} / z 123$ is ${ }^{63} \mathrm{CuC}_{5}^{-}$and at $m / z 125$ is ${ }^{65} \mathrm{CuC}_{5}^{-}$.

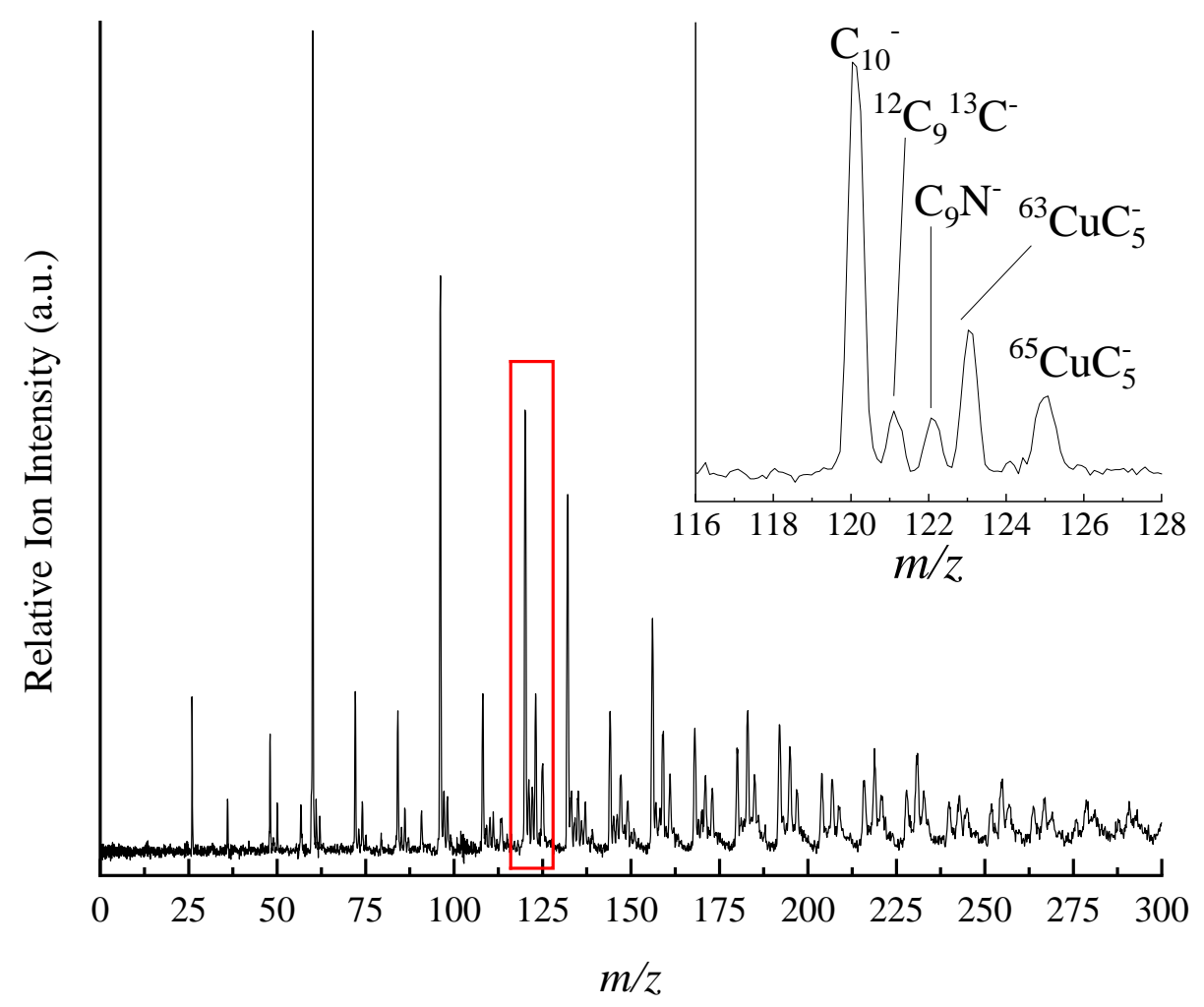


Figure S4. Mass spectra for $\mathrm{GF}+\mathrm{NaNO}_{3}$ reaction from Figure 6(d), separated and shown over full $\mathrm{m} / \mathrm{z}$, range

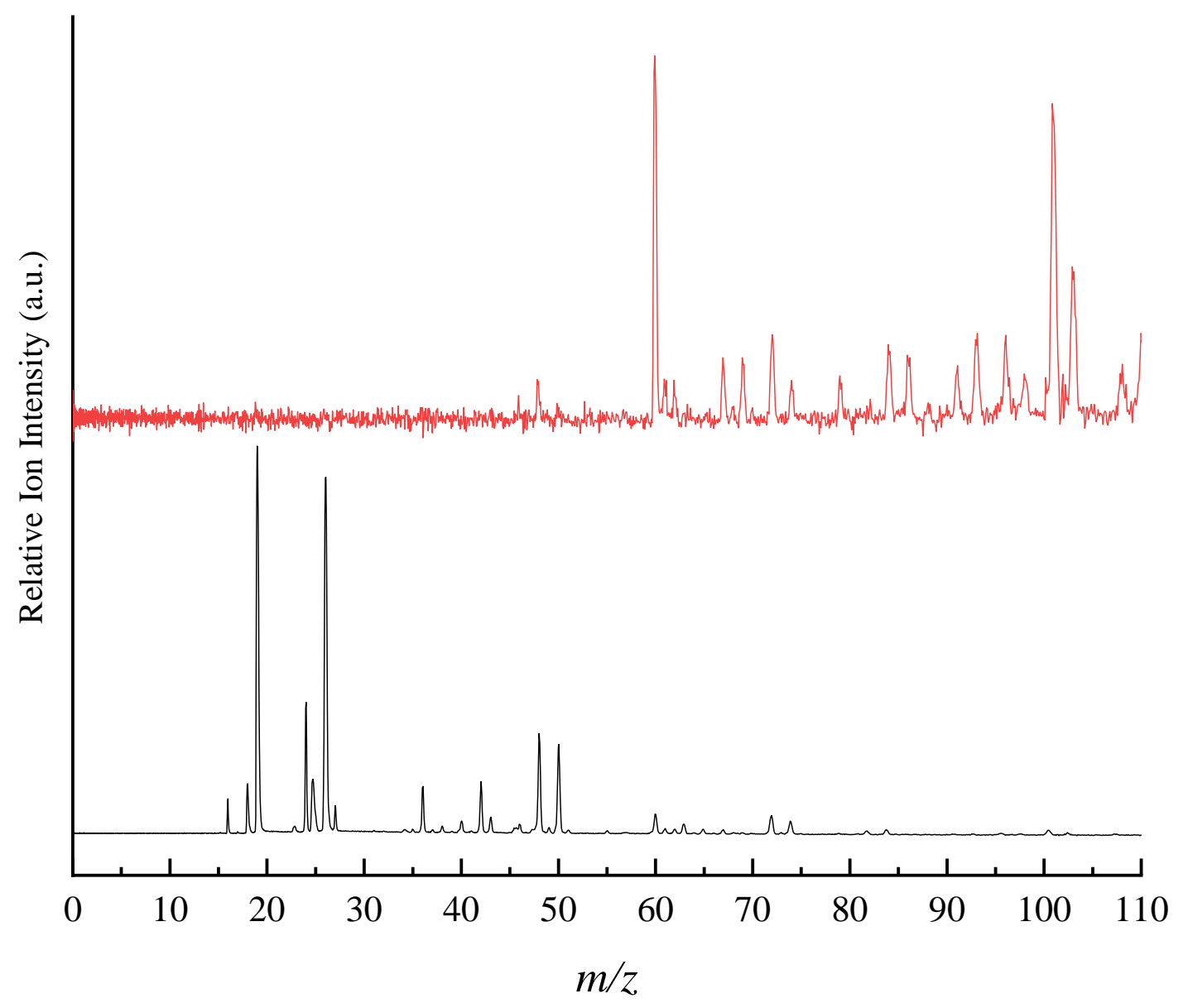


Laser desorption/ionization mass spectrometric analysis. In the LDI mass spectra for $\mathrm{C}_{3} \mathrm{~N}_{4}$, there are several overlapping patterns that emerge. The five prominent patterns are peaks separated by $25 \mathrm{~m} / \mathrm{z}\left(\mathrm{C}_{2} \mathrm{H}\right), 42 \mathrm{~m} / \mathrm{z}\left(\mathrm{N}_{3}\right), 50 \mathrm{~m} / \mathrm{z}$ $\left(\mathrm{C}_{3} \mathrm{~N}\right), 67 \mathrm{~m} / z\left(\mathrm{C}_{2} \mathrm{~N}_{3} \mathrm{H}\right)$, and $92 \mathrm{~m} / z\left(\mathrm{C}_{3} \mathrm{~N}_{4}\right)$. Only a representative set of peaks for each mass pattern are labeled in $\mathrm{S} 9$ and S10, even though there are several overlapping sets. The tables of all overlapping mass patterns for the major peaks are included in S11 and S12. In S9, the most prominent peak is $\mathrm{C}_{2} \mathrm{~N}_{3}$ at $66 \mathrm{~m} / z$. (Tables of possible stoichiometries for anion and cation peaks are given in S13 and S14). There is a series of peaks separated by $25 \mathrm{~m} / \mathrm{z}$ from $150 \mathrm{~m} / \mathrm{z}$ to $250 \mathrm{~m} / \mathrm{z}$. There are two short series of peaks separated by $42 \mathrm{~m} / \mathrm{z}$, at $158-242 \mathrm{~m} / \mathrm{z}$ and $317-401 \mathrm{~m} / \mathrm{z}$. The peaks at 242, 292, and 342 $\mathrm{m} / \mathrm{z}$ are separated by $50 \mathrm{~m} / \mathrm{z}$, as well as the endpoints and middle of the $25 \mathrm{~m} / \mathrm{z}$ series, 150,200 , and $250 \mathrm{~m} / \mathrm{z}$. The series of peaks separated by $67 \mathrm{~m} / \mathrm{z}$ begins with the intense $66 \mathrm{~m} / z$ peak and concludes with the small $401 \mathrm{~m} / \mathrm{z}$ peak. Finally, the peaks at 108, 200, 292, and $384 \mathrm{~m} / \mathrm{z}$ constitute one of the series separated by $92 \mathrm{~m} / \mathrm{z}$. In S10, the prominent peak at $\mathrm{m} / \mathrm{z}$ 244 could have any of three stoichiometries, though the stoichiometries $\mathrm{C}_{11} \mathrm{~N}_{8}$ and $\mathrm{C}_{4} \mathrm{~N}_{14}$ are more likely since the starting material has the formula $\mathrm{C}_{3} \mathrm{~N}_{4}$.

These five masses were chosen based on a histogram of the peak spacings. For both the cations and anions, there were many different mass spacings, but these were the ones which occurred most frequently. Often, cluster ions are more stable as either anions or cations, but here we see five species which seem to be stable in both modes. It is possible that the masses correspond to neutral species. Regardless, the number of overlapping mass series in both mass spectra is remarkable. We do not see many peaks in S9 and S10 that are also present in the mass spectra measured using direct laser ablation. This is likely due to the higher nitrogen content in carbon nitride which allows us to see a greater number of stoichiometric or nitrogen-rich clusters. 
Figure S5. Mass spectrum for anionic species generated from matrix-free LDI of $\mathrm{C}_{3} \mathrm{~N}_{4}$. Symbols are used to show patterns of peaks separated by given masses. Peaks separated by $25 \mathrm{~m} / \mathrm{z}$ are indicated by *, $42 \mathrm{~m} / \mathrm{z}$ by,$+ 50 \mathrm{~m} / \mathrm{z}$ by $\boldsymbol{\nabla}, 67 \mathrm{~m} / \mathrm{z}$ by $\bullet$, and $92 \mathrm{~m} / \mathrm{z}$ by $\bullet$. Symbols of two different colors indicate two separate (discontinuous) series of peaks separated by the same $\mathrm{m} / z$.

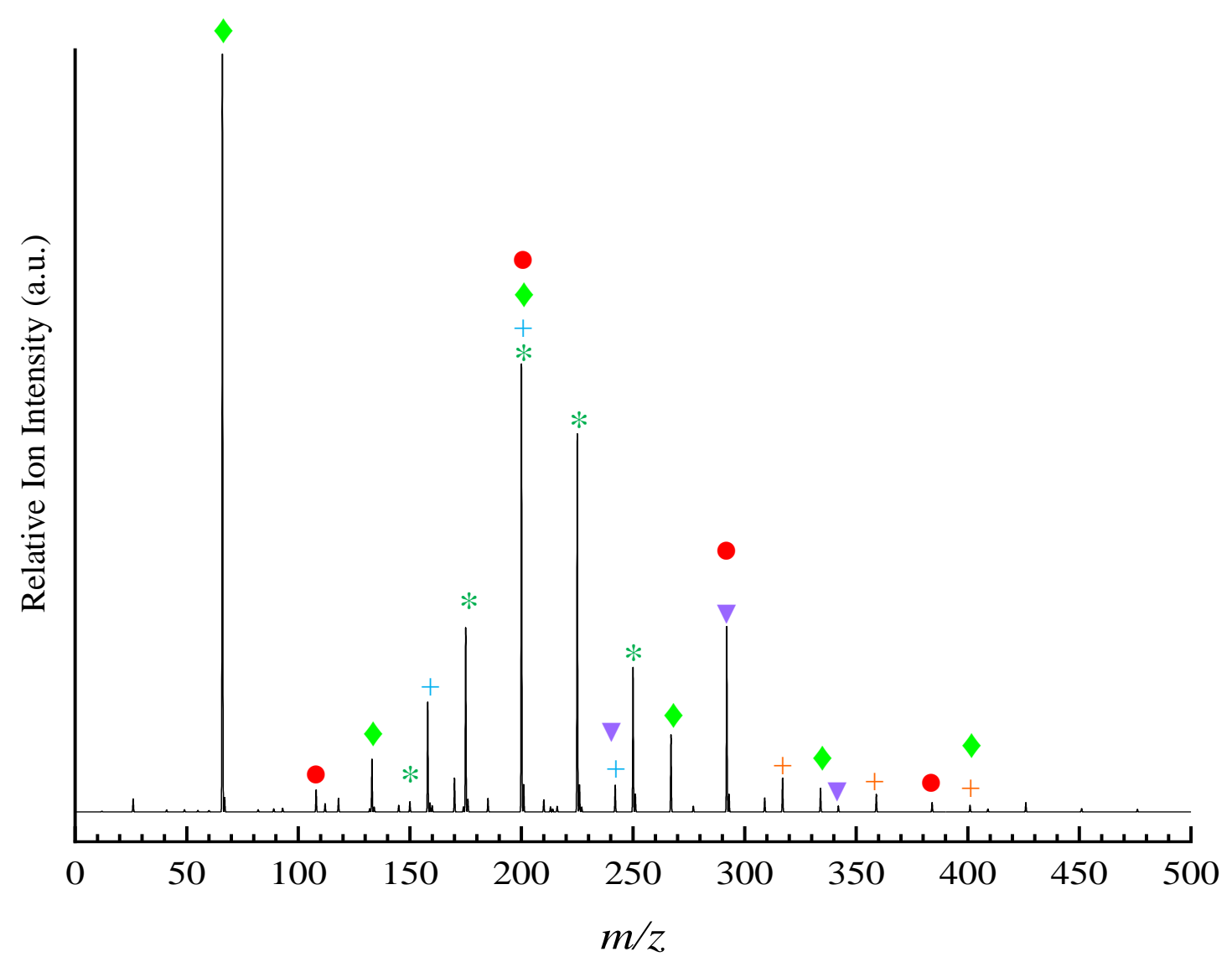


Figure S6. Mass spectrum for cationic species generated from matrix-free LDI of $\mathrm{C}_{3} \mathrm{~N}_{4}$. Symbols are used to show patterns of peaks separated by given masses. Peaks separated by $25 \mathrm{~m} / z$ are indicated by *, $42 \mathrm{~m} / \mathrm{z}$ by,$+ 50 \mathrm{~m} / \mathrm{z}$ by $\boldsymbol{\nabla}, 67 \mathrm{~m} / \mathrm{z}$ by $\bullet$, and $92 \mathrm{~m} / \mathrm{z}$ by $\bullet$. Symbols of two different colors indicate two separate (discontinuous) series of peaks separated by the same $\mathrm{m} / \mathrm{z}$.

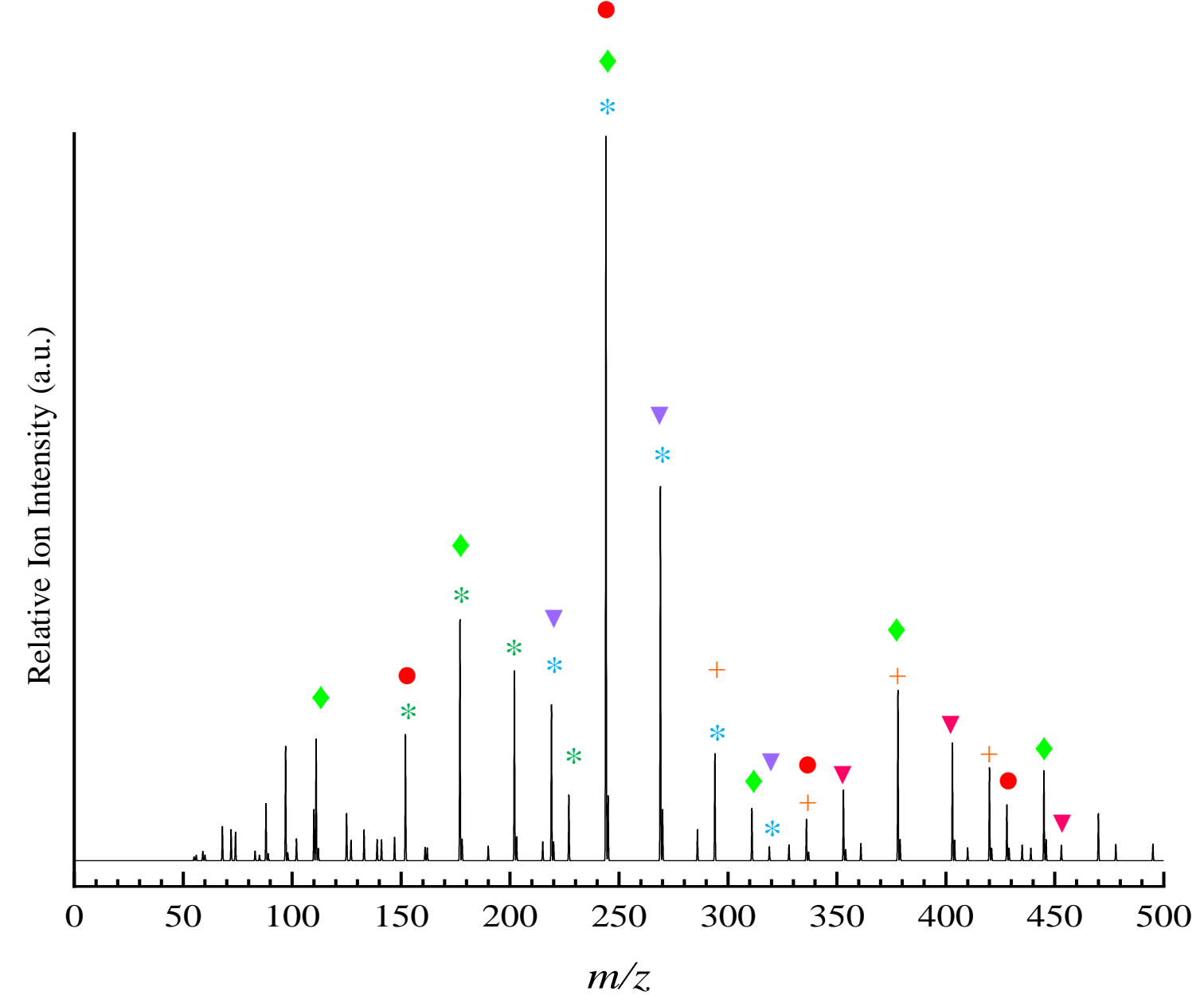


Table S8. Columns showing the series of mass separations for the major peaks seen in Figure S5. Each color in a given column indicates a single set of peaks separated by the mass value given at the top of the column. Any white spaces indicate that the given peak does not fit the mass pattern for that column.

\begin{tabular}{|c|}
\hline 25 \\
\hline 66 \\
\hline 108 \\
133 \\
150 \\
\hline 158 \\
175 \\
200 \\
225 \\
\hline 242 \\
\hline 250 \\
267 \\
292 \\
309 \\
317 \\
334 \\
342 \\
359 \\
384 \\
401 \\
426 \\
\hline
\end{tabular}

\begin{tabular}{|c|}
\hline 42 \\
\hline 66 \\
108 \\
133 \\
150 \\
158 \\
175 \\
\hline 200 \\
225 \\
242 \\
250 \\
267 \\
292 \\
309 \\
\hline 317 \\
\hline 334 \\
\hline 342 \\
\hline 359 \\
\hline 384 \\
\hline 401 \\
\hline 426 \\
\hline
\end{tabular}

\begin{tabular}{|c|}
\hline 50 \\
\hline 66 \\
\hline 108 \\
\hline 133 \\
\hline 150 \\
\hline 158 \\
\hline 175 \\
\hline 200 \\
\hline 225 \\
242 \\
\hline 250 \\
\hline 267 \\
292 \\
\hline 309 \\
\hline 317 \\
\hline 334 \\
\hline 342 \\
\hline 359 \\
\hline 384 \\
\hline 401 \\
426 \\
\hline
\end{tabular}

\begin{tabular}{|c|}
\hline 67 \\
\hline 66 \\
\hline 108 \\
\hline 133 \\
\hline 150 \\
\hline 158 \\
\hline 175 \\
\hline 200 \\
\hline 225 \\
\hline 242 \\
\hline 250 \\
\hline 267 \\
\hline 292 \\
\hline 309 \\
\hline 317 \\
\hline 334 \\
\hline 342 \\
\hline 359 \\
\hline 384 \\
\hline 401 \\
\hline 426 \\
\hline
\end{tabular}

\begin{tabular}{|c|}
\hline 92 \\
\hline 66 \\
\hline 108 \\
133 \\
150 \\
\hline 158 \\
\hline 175 \\
\hline 200 \\
\hline 225 \\
242 \\
\hline 250 \\
\hline 267 \\
\hline 292 \\
\hline 309 \\
\hline 317 \\
\hline 334 \\
\hline 342 \\
\hline 359 \\
\hline 384 \\
\hline 401 \\
\hline 426 \\
\hline
\end{tabular}


Table S9. Columns showing the series of mass separations for the major peaks seen in Figure S6. Each color in a given column indicates a single set of peaks separated by the mass value given at the top of the column. Any white spaces indicate that the given peak does not fit the mass pattern for that column.

\begin{tabular}{|c|}
\hline $\mathbf{2 5}$ \\
\hline 110 \\
\hline 152 \\
177 \\
202 \\
\hline 219 \\
\hline 227 \\
\hline 244 \\
269 \\
\hline 286 \\
\hline 294 \\
311 \\
319 \\
336 \\
353 \\
361 \\
378 \\
403 \\
420 \\
428 \\
445 \\
453 \\
470 \\
478 \\
495 \\
\hline
\end{tabular}

\begin{tabular}{|c|}
\hline 42 \\
\hline 110 \\
152 \\
177 \\
202 \\
219 \\
\hline 227 \\
244 \\
269 \\
286 \\
294 \\
311 \\
319 \\
336 \\
353 \\
\hline 361 \\
378 \\
403 \\
420 \\
428 \\
445 \\
453 \\
470 \\
\hline 478 \\
495 \\
\hline
\end{tabular}

\begin{tabular}{|c|}
\hline $\mathbf{5 0}$ \\
\hline 110 \\
\hline 152 \\
\hline 177 \\
\hline 202 \\
\hline 219 \\
\hline 227 \\
\hline 244 \\
269 \\
286 \\
294 \\
311 \\
\hline 319 \\
336 \\
\hline 353 \\
\hline 361 \\
\hline 378 \\
\hline 403 \\
\hline 420 \\
\hline 428 \\
445 \\
\hline 453 \\
\hline 470 \\
\hline 478 \\
495 \\
\hline
\end{tabular}

\begin{tabular}{|c|}
\hline 67 \\
\hline 110 \\
\hline 152 \\
\hline 177 \\
\hline 202 \\
\hline 219 \\
\hline 227 \\
\hline 244 \\
\hline 269 \\
\hline 286 \\
\hline 294 \\
\hline 311 \\
\hline 319 \\
\hline 336 \\
\hline 353 \\
\hline 361 \\
\hline 378 \\
\hline 403 \\
\hline 420 \\
\hline 428 \\
\hline 445 \\
\hline 453 \\
\hline 470 \\
\hline 478 \\
\hline 495 \\
\hline
\end{tabular}

\begin{tabular}{|c|}
\hline 92 \\
\hline 110 \\
\hline 152 \\
\hline 202 \\
\hline 219 \\
227 \\
\hline 244 \\
\hline 269 \\
\hline 286 \\
\hline 294 \\
\hline 311 \\
\hline 319 \\
\hline 336 \\
\hline 353 \\
\hline 361 \\
\hline 378 \\
\hline 403 \\
\hline 420 \\
\hline 428 \\
\hline 445 \\
\hline 453 \\
\hline 470 \\
\hline 478 \\
\hline 495 \\
\hline
\end{tabular}


Table S10. List of all masses observed in Figure S5, along with likely assignments

\begin{tabular}{|c|c|c|c|c|c|c|c|c|c|c|c|}
\hline \multirow{2}{*}{$\begin{array}{c}m / z \\
12\end{array}$} & \multicolumn{3}{|c|}{ Assignments } & \multirow{2}{*}{$\begin{array}{l}m / z \\
150\end{array}$} & \multicolumn{3}{|c|}{ Assignments } & \multirow{2}{*}{$\begin{array}{l}m / z \\
242\end{array}$} & \multicolumn{3}{|c|}{ Assignments } \\
\hline & C & & & & $\mathrm{C}_{9} \mathrm{~N}_{3}$ & $\mathrm{C}_{10} \mathrm{~N}_{2} \mathrm{H}_{2}$ & $\mathrm{C}_{2} \mathrm{~N}_{9}$ & & $\mathrm{C}_{12} \mathrm{~N}_{7}$ & $\mathrm{C}_{5} \mathrm{~N}_{13}$ & $\mathrm{C}_{19} \mathrm{~N}$ \\
\hline 26 & $\mathrm{CN}$ & & & 158 & $\mathrm{C}_{5} \mathrm{~N}_{7}$ & $\mathrm{C}_{6} \mathrm{~N}_{6} \mathrm{H}_{2}$ & $\mathrm{C}_{12} \mathrm{~N}$ & 250 & $\mathrm{C}_{8} \mathrm{~N}_{11}$ & $\mathrm{C}_{15} \mathrm{~N}_{5}$ & \\
\hline 41 & $\mathrm{CN}_{2} \mathrm{H}$ & & & 159 & $\mathrm{C}_{5} \mathrm{~N}_{7} \mathrm{H}$ & $\mathrm{C}_{6} \mathrm{~N}_{6} \mathrm{H}_{3}$ & $\mathrm{C}_{12} \mathrm{NH}$ & 251 & $\mathrm{C}_{8} \mathrm{~N}_{11} \mathrm{H}$ & $\mathrm{C}_{15} \mathrm{~N}_{5} \mathrm{H}$ & \\
\hline 49 & $\mathrm{C}_{4} \mathrm{H}$ & & & 160 & $\mathrm{C}_{4} \mathrm{~N}_{8}$ & $\mathrm{C}_{11} \mathrm{~N}_{2}$ & & 267 & $\mathrm{C}_{7} \mathrm{~N}_{13} \mathrm{H}$ & $\mathrm{C}_{14} \mathrm{~N}_{7} \mathrm{H}$ & $\mathrm{C}_{21} \mathrm{NH}$ \\
\hline 55 & $\mathrm{CN}_{3} \mathrm{H}$ & & & 170 & $\mathrm{C}_{6} \mathrm{~N}_{7}$ & $\mathrm{C}_{7} \mathrm{~N}_{6} \mathrm{H}_{2}$ & $\mathrm{C}_{13} \mathrm{~N}$ & 277 & $\mathrm{C}_{9} \mathrm{~N}_{12} \mathrm{H}$ & $\mathrm{C}_{16} \mathrm{~N}_{6} \mathrm{H}$ & $\mathrm{C}_{23} \mathrm{H}$ \\
\hline 60 & $C_{5}$ & & & 174 & $\mathrm{C}_{11} \mathrm{~N}_{3}$ & $\mathrm{C}_{12} \mathrm{~N}_{2} \mathrm{H}_{2}$ & $\mathrm{C}_{4} \mathrm{~N}_{9}$ & 292 & $\mathrm{C}_{8} \mathrm{~N}_{14}$ & $\mathrm{C}_{15} \mathrm{~N}_{8}$ & $\mathrm{C}_{22} \mathrm{~N}_{2}$ \\
\hline 66 & $\mathrm{C}_{2} \mathrm{~N}_{3}$ & $\mathrm{C}_{3} \mathrm{~N}_{2} \mathrm{H}_{2}$ & & 175 & $\mathrm{C}_{11} \mathrm{~N}_{3} \mathrm{H}$ & $\mathrm{C}_{12} \mathrm{~N}_{2} \mathrm{H}_{3}$ & $\mathrm{C}_{4} \mathrm{~N}_{9} \mathrm{H}$ & 293 & $\mathrm{C}_{8} \mathrm{~N}_{14} \mathrm{H}$ & $\mathrm{C}_{15} \mathrm{~N}_{8} \mathrm{H}$ & $\mathrm{C}_{22} \mathrm{~N}_{2} \mathrm{H}$ \\
\hline 67 & $\mathrm{C}_{2} \mathrm{~N}_{3} \mathrm{H}$ & & & 176 & $\mathrm{C}_{10} \mathrm{~N}_{4}$ & $\mathrm{C}_{11} \mathrm{~N}_{3} \mathrm{H}_{2}$ & $\mathrm{C}_{3} \mathrm{~N}_{10}$ & 309 & $\mathrm{C}_{7} \mathrm{~N}_{16} \mathrm{H}$ & $\mathrm{C}_{14} \mathrm{~N}_{10} \mathrm{H}$ & $\mathrm{C}_{21} \mathrm{~N}_{4} \mathrm{H}$ \\
\hline 82 & $\mathrm{C}_{2} \mathrm{~N}_{4} \mathrm{H}_{2}$ & $\mathrm{C}_{4} \mathrm{~N}_{2} \mathrm{H}_{6}$ & $\mathrm{C}_{3} \mathrm{~N}_{3} \mathrm{H}_{4}$ & 185 & $\mathrm{C}_{6} \mathrm{~N}_{8} \mathrm{H}$ & $\mathrm{C}_{13} \mathrm{~N}_{2} \mathrm{H}$ & & 317 & $\mathrm{C}_{10} \mathrm{~N}_{14} \mathrm{H}$ & $\mathrm{C}_{17} \mathrm{~N}_{8} \mathrm{H}$ & $\mathrm{C}_{24} \mathrm{~N}_{2} \mathrm{H}$ \\
\hline 89 & $\mathrm{C}_{5} \mathrm{~N}_{2} \mathrm{H}$ & & & 200 & $\mathrm{C}_{5} \mathrm{~N}_{10}$ & $\mathrm{C}_{12} \mathrm{~N}_{4}$ & & 334 & $\mathrm{C}_{8} \mathrm{~N}_{17}$ & $\mathrm{C}_{15} \mathrm{~N}_{11}$ & $\mathrm{C}_{22} \mathrm{~N}_{5}$ \\
\hline 93 & $\mathrm{C}_{3} \mathrm{~N}_{4} \mathrm{H}$ & & & 201 & $\mathrm{C}_{5} \mathrm{~N}_{10} \mathrm{H}$ & $\mathrm{C}_{12} \mathrm{~N}_{4} \mathrm{H}$ & & 342 & $\mathrm{C}_{11} \mathrm{~N}_{15}$ & $\mathrm{C}_{18} \mathrm{~N}_{9}$ & $\mathrm{C}_{25} \mathrm{~N}_{3}$ \\
\hline 108 & $\mathrm{C}_{9}$ & $\mathrm{C}_{2} \mathrm{~N}_{6}$ & $\mathrm{C}_{3} \mathrm{~N}_{5} \mathrm{H}_{2}$ & 210 & $\mathrm{C}_{7} \mathrm{~N}_{9}$ & $\mathrm{C}_{14} \mathrm{~N}_{3}$ & & 359 & $\mathrm{C}_{10} \mathrm{~N}_{17} \mathrm{H}$ & $\mathrm{C}_{17} \mathrm{~N}_{11} \mathrm{H}$ & $\mathrm{C}_{24} \mathrm{~N}_{5} \mathrm{H}$ \\
\hline 112 & $\mathrm{C}_{7} \mathrm{~N}_{2}$ & & & 213 & $\mathrm{C}_{6} \mathrm{~N}_{10} \mathrm{H}$ & $\mathrm{C}_{13} \mathrm{~N}_{4} \mathrm{H}$ & & 384 & $\mathrm{C}_{11} \mathrm{~N}_{18}$ & $\mathrm{C}_{18} \mathrm{~N}_{12}$ & $\mathrm{C}_{25} \mathrm{~N}_{6}$ \\
\hline 118 & $\mathrm{C}_{4} \mathrm{~N}_{5}$ & $\mathrm{C}_{5} \mathrm{~N}_{4} \mathrm{H}_{2}$ & & 214 & $\mathrm{C}_{5} \mathrm{~N}_{11}$ & $\mathrm{C}_{12} \mathrm{~N}_{5}$ & & 401 & $\mathrm{C}_{10} \mathrm{~N}_{20} \mathrm{H}$ & $\mathrm{C}_{17} \mathrm{~N}_{14} \mathrm{H}$ & $\mathrm{C}_{31} \mathrm{~N}_{2} \mathrm{H}$ \\
\hline 132 & $\mathrm{C}_{4} \mathrm{~N}_{6}$ & $\mathrm{C}_{11}$ & $\mathrm{C}_{5} \mathrm{~N}_{5} \mathrm{H}_{2}$ & 216 & $\mathrm{C}_{4} \mathrm{~N}_{12}$ & $\mathrm{C}_{11} \mathrm{~N}_{6}$ & $\mathrm{C}_{18}$ & 409 & $\mathrm{C}_{13} \mathrm{~N}_{18} \mathrm{H}$ & $\mathrm{C}_{20} \mathrm{~N}_{12} \mathrm{H}$ & $\mathrm{C}_{27} \mathrm{~N}_{6} \mathrm{H}$ \\
\hline 133 & $\mathrm{C}_{4} \mathrm{~N}_{6} \mathrm{H}$ & $\mathrm{C}_{11} \mathrm{H}$ & $\mathrm{C}_{5} \mathrm{~N}_{5} \mathrm{H}_{3}$ & 225 & $\mathrm{C}_{7} \mathrm{~N}_{10} \mathrm{H}$ & $\mathrm{C}_{14} \mathrm{~N}_{4} \mathrm{H}$ & & 426 & $\mathrm{C}_{11} \mathrm{~N}_{21}$ & $\mathrm{C}_{18} \mathrm{~N}_{15}$ & $\mathrm{C}_{32} \mathrm{~N}_{3}$ \\
\hline 134 & $\mathrm{C}_{4} \mathrm{~N}_{6} \mathrm{H}_{2}$ & $\mathrm{C}_{10} \mathrm{~N}$ & $\mathrm{C}_{3} \mathrm{~N}_{7}$ & 226 & $\mathrm{C}_{6} \mathrm{~N}_{11}$ & $\mathrm{C}_{13} \mathrm{~N}_{5}$ & & 451 & $\mathrm{C}_{13} \mathrm{~N}_{21} \mathrm{H}$ & $\mathrm{C}_{20} \mathrm{~N}_{15} \mathrm{H}$ & $\mathrm{C}_{34} \mathrm{~N}_{3} \mathrm{H}$ \\
\hline 145 & $\mathrm{C}_{5} \mathrm{~N}_{6} \mathrm{H}$ & $\mathrm{C}_{12} \mathrm{H}$ & & 227 & $\mathrm{C}_{6} \mathrm{~N}_{11} \mathrm{H}$ & $\mathrm{C}_{13} \mathrm{~N}_{5} \mathrm{H}$ & & 476 & $\mathrm{C}_{14} \mathrm{~N}_{22}$ & $\mathrm{C}_{21} \mathrm{~N}_{16}$ & $\mathrm{C}_{35} \mathrm{~N}_{4}$ \\
\hline
\end{tabular}


Table S11. List of all masses observed in Figure S6, along with likely assignments

\begin{tabular}{|c|c|c|c|c|c|c|c|c|c|c|c|c|}
\hline \multirow{2}{*}{$\begin{array}{c}m / z \\
39\end{array}$} & \multicolumn{3}{|c|}{ Assignments } & \multirow{2}{*}{$\begin{array}{l}m / z \\
141\end{array}$} & \multicolumn{3}{|c|}{ Assignments } & \multirow{2}{*}{$\begin{array}{l}m / z \\
336\end{array}$} & \multicolumn{4}{|c|}{ Assignments } \\
\hline & $\mathrm{C}_{2} \mathrm{NH}$ & & & & $\mathrm{C}_{7} \mathrm{~N}_{4} \mathrm{H}$ & & & & $\mathrm{C}_{21} \mathrm{~N}_{6}$ & $\mathrm{C}_{14} \mathrm{~N}_{12}$ & $\mathrm{C}_{7} \mathrm{~N}_{18}$ & \\
\hline 55 & $\mathrm{CN}_{3} \mathrm{H}$ & & & 147 & $\mathrm{C}_{11} \mathrm{NH}$ & $\mathrm{C}_{4} \mathrm{~N}_{7} \mathrm{H}$ & & 337 & $\mathrm{C}_{21} \mathrm{~N}_{6} \mathrm{H}$ & $\mathrm{C}_{14} \mathrm{~N}_{12} \mathrm{H}$ & $\mathrm{C}_{7} \mathrm{~N}_{18} \mathrm{H}$ & \\
\hline 56 & $\mathrm{CN}_{3} \mathrm{H}_{2}$ & & & 152 & $\mathrm{C}_{8} \mathrm{~N}_{4}$ & $\mathrm{C}_{9} \mathrm{~N}_{3} \mathrm{H}_{2}$ & & 353 & $\mathrm{C}_{20} \mathrm{~N}_{8} \mathrm{H}$ & $\mathrm{C}_{13} \mathrm{~N}_{14} \mathrm{H}$ & $\mathrm{C}_{6} \mathrm{~N}_{20} \mathrm{H}$ & \\
\hline 59 & $\mathrm{CN}_{3} \mathrm{H}_{5}$ & & & 161 & $\mathrm{C}_{11} \mathrm{~N}_{2} \mathrm{H}$ & $\mathrm{C}_{4} \mathrm{~N}_{8} \mathrm{H}$ & & 354 & $\mathrm{C}_{26} \mathrm{~N}_{3}$ & $\mathrm{C}_{19} \mathrm{~N}_{9}$ & $\mathrm{C}_{12} \mathrm{~N}_{15}$ & \\
\hline 60 & $C_{5}$ & & & 162 & $\mathrm{C}_{10} \mathrm{~N}_{3}$ & $\mathrm{C}_{3} \mathrm{~N}_{9}$ & & 361 & $\mathrm{C}_{23} \mathrm{~N}_{6} \mathrm{H}$ & $\mathrm{C}_{16} \mathrm{~N}_{12} \mathrm{H}$ & $\mathrm{C}_{9} \mathrm{~N}_{18} \mathrm{H}$ & \\
\hline 68 & $\mathrm{CN}_{4}$ & $\mathrm{C}_{2} \mathrm{~N}_{3} \mathrm{H}_{2}$ & $\mathrm{C}_{3} \mathrm{~N}_{2} \mathrm{H}_{4}$ & 177 & $\mathrm{C}_{10} \mathrm{~N}_{4} \mathrm{H}$ & $\mathrm{C}_{3} \mathrm{~N}_{10} \mathrm{H}$ & & 378 & $\mathrm{C}_{28} \mathrm{~N}_{3}$ & $\mathrm{C}_{21} \mathrm{~N}_{9}$ & $\mathrm{C}_{14} \mathrm{~N}_{15}$ & $\mathrm{C}_{7} \mathrm{~N}_{21}$ \\
\hline 72 & $\mathrm{C}_{6}$ & & & 178 & $\mathrm{C}_{9} \mathrm{~N}_{5}$ & $\mathrm{C}_{3} \mathrm{~N}_{10} \mathrm{H}_{2}$ & & 379 & $\mathrm{C}_{28} \mathrm{~N}_{3} \mathrm{H}$ & $\mathrm{C}_{21} \mathrm{~N}_{9} \mathrm{H}$ & $\mathrm{C}_{14} \mathrm{~N}_{15} \mathrm{H}$ & $\mathrm{C}_{7} \mathrm{~N}_{21} \mathrm{H}$ \\
\hline 74 & $\mathrm{C}_{5} \mathrm{~N}$ & & & 190 & $\mathrm{C}_{10} \mathrm{~N}_{5}$ & $\mathrm{C}_{3} \mathrm{~N}_{11}$ & & 403 & $\mathrm{C}_{30} \mathrm{~N}_{3} \mathrm{H}$ & $\mathrm{C}_{23} \mathrm{~N}_{9} \mathrm{H}$ & $\mathrm{C}_{16} \mathrm{~N}_{15} \mathrm{H}$ & $\mathrm{C}_{9} \mathrm{~N}_{21} \mathrm{H}$ \\
\hline 83 & $\mathrm{CN}_{5} \mathrm{H}$ & $\mathrm{C}_{2} \mathrm{~N}_{4} \mathrm{H}_{3}$ & $\mathrm{C}_{3} \mathrm{~N}_{3} \mathrm{H}_{5}$ & 202 & $\mathrm{C}_{11} \mathrm{~N}_{5}$ & $\mathrm{C}_{4} \mathrm{~N}_{11}$ & & 404 & $\mathrm{C}_{29} \mathrm{~N}_{4}$ & $\mathrm{C}_{22} \mathrm{~N}_{10}$ & $\mathrm{C}_{15} \mathrm{~N}_{16}$ & $\mathrm{C}_{8} \mathrm{~N}_{22}$ \\
\hline 85 & $\mathrm{CN}_{5} \mathrm{H}_{3}$ & $\mathrm{C}_{7} \mathrm{H}$ & & 203 & $\mathrm{C}_{11} \mathrm{~N}_{5} \mathrm{H}$ & $\mathrm{C}_{4} \mathrm{~N}_{11} \mathrm{H}$ & & 410 & $\mathrm{C}_{33} \mathrm{~N}$ & $\mathrm{C}_{26} \mathrm{~N}_{7}$ & $\mathrm{C}_{19} \mathrm{~N}_{13}$ & $\mathrm{C}_{12} \mathrm{~N}_{19}$ \\
\hline 88 & $\mathrm{C}_{5} \mathrm{~N}_{2}$ & $\mathrm{C}_{6} \mathrm{NH}_{2}$ & & 215 & $\mathrm{C}_{12} \mathrm{~N}_{5} \mathrm{H}$ & $\mathrm{C}_{5} \mathrm{~N}_{11} \mathrm{H}$ & & 420 & $\mathrm{C}_{28} \mathrm{~N}_{6}$ & $\mathrm{C}_{21} \mathrm{~N}_{12}$ & $\mathrm{C}_{14} \mathrm{~N}_{18}$ & $\mathrm{C}_{7} \mathrm{~N}_{24}$ \\
\hline 89 & $\mathrm{C}_{5} \mathrm{~N}_{2} \mathrm{H}$ & $\mathrm{C}_{6} \mathrm{NH}_{3}$ & & 219 & $\mathrm{C}_{17} \mathrm{NH}$ & $\mathrm{C}_{10} \mathrm{~N}_{7} \mathrm{H}$ & $\mathrm{C}_{3} \mathrm{~N}_{13} \mathrm{H}$ & 421 & $\mathrm{C}_{28} \mathrm{~N}_{6} \mathrm{H}$ & $\mathrm{C}_{21} \mathrm{~N}_{12} \mathrm{H}$ & $\mathrm{C}_{14} \mathrm{~N}_{18} \mathrm{H}$ & $\mathrm{C}_{7} \mathrm{~N}_{24} \mathrm{H}$ \\
\hline 97 & $\mathrm{C}_{8} \mathrm{H}$ & $\mathrm{C}_{2} \mathrm{~N}_{5} \mathrm{H}_{3}$ & & 220 & $\mathrm{C}_{16} \mathrm{~N}_{2}$ & $\mathrm{C}_{9} \mathrm{~N}_{8}$ & & 428 & $\mathrm{C}_{31} \mathrm{~N}_{4}$ & $\mathrm{C}_{24} \mathrm{~N}_{10}$ & $\mathrm{C}_{17} \mathrm{~N}_{16}$ & $\mathrm{C}_{10} \mathrm{~N}_{22}$ \\
\hline 98 & $\mathrm{C}_{7} \mathrm{~N}$ & & & 227 & $\mathrm{C}_{13} \mathrm{~N}_{5} \mathrm{H}$ & $\mathrm{C}_{6} \mathrm{~N}_{11} \mathrm{H}$ & & 429 & $\mathrm{C}_{31} \mathrm{~N}_{4} \mathrm{H}$ & $\mathrm{C}_{24} \mathrm{~N}_{10} \mathrm{H}$ & $\mathrm{C}_{17} \mathrm{~N}_{16} \mathrm{H}$ & $\mathrm{C}_{10} \mathrm{~N}_{22} \mathrm{H}$ \\
\hline 102 & $\mathrm{C}_{5} \mathrm{~N}_{3}$ & & & 244 & $\mathrm{C}_{18} \mathrm{~N}_{2}$ & $\mathrm{C}_{11} \mathrm{~N}_{8}$ & $\mathrm{C}_{4} \mathrm{~N}_{14}$ & 435 & $\mathrm{C}_{28} \mathrm{~N}_{7} \mathrm{H}$ & $\mathrm{C}_{21} \mathrm{~N}_{13} \mathrm{H}$ & $\mathrm{C}_{14} \mathrm{~N}_{19} \mathrm{H}$ & $\mathrm{C}_{7} \mathrm{~N}_{25} \mathrm{H}$ \\
\hline 110 & $\mathrm{C}_{8} \mathrm{~N}$ & & & 245 & $\mathrm{C}_{18} \mathrm{~N}_{2} \mathrm{H}$ & $\mathrm{C}_{11} \mathrm{~N}_{8} \mathrm{H}$ & $\mathrm{C}_{4} \mathrm{~N}_{14} \mathrm{H}$ & 439 & $\mathrm{C}_{33} \mathrm{~N}_{3} \mathrm{H}$ & $\mathrm{C}_{26} \mathrm{~N}_{9} \mathrm{H}$ & $\mathrm{C}_{19} \mathrm{~N}_{15} \mathrm{H}$ & $\mathrm{C}_{12} \mathrm{~N}_{21} \mathrm{H}$ \\
\hline 111 & $\mathrm{C}_{8} \mathrm{NH}$ & & & 269 & $\mathrm{C}_{20} \mathrm{~N}_{2} \mathrm{H}$ & $\mathrm{C}_{13} \mathrm{~N}_{8} \mathrm{H}$ & $\mathrm{C}_{6} \mathrm{~N}_{14} \mathrm{H}$ & 445 & $\mathrm{C}_{30} \mathrm{~N}_{6} \mathrm{H}$ & $\mathrm{C}_{23} \mathrm{~N}_{12} \mathrm{H}$ & $\mathrm{C}_{16} \mathrm{~N}_{18} \mathrm{H}$ & $\mathrm{C}_{9} \mathrm{~N}_{24} \mathrm{H}$ \\
\hline 112 & $\mathrm{C}_{7} \mathrm{~N}_{2}$ & $\mathrm{C}_{8} \mathrm{NH}_{2}$ & & 270 & $\mathrm{C}_{19} \mathrm{~N}_{3}$ & $\mathrm{C}_{12} \mathrm{~N}_{9}$ & $\mathrm{C}_{5} \mathrm{~N}_{15}$ & 446 & $\mathrm{C}_{29} \mathrm{~N}_{7}$ & $\mathrm{C}_{22} \mathrm{~N}_{13}$ & $\mathrm{C}_{15} \mathrm{~N}_{19}$ & $\mathrm{C}_{8} \mathrm{~N}_{25}$ \\
\hline 125 & $\mathrm{C}_{8} \mathrm{~N}_{2} \mathrm{H}$ & & & 286 & $\mathrm{C}_{18} \mathrm{~N}_{5}$ & $\mathrm{C}_{11} \mathrm{~N}_{11}$ & $\mathrm{C}_{4} \mathrm{~N}_{17}$ & 453 & $\mathrm{C}_{33} \mathrm{~N}_{4} \mathrm{H}$ & $\mathrm{C}_{26} \mathrm{~N}_{10} \mathrm{H}$ & $\mathrm{C}_{19} \mathrm{~N}_{16} \mathrm{H}$ & $\mathrm{C}_{12} \mathrm{~N}_{22} \mathrm{H}$ \\
\hline 127 & $\mathrm{C}_{7} \mathrm{~N}_{3} \mathrm{H}$ & & & 294 & $\mathrm{C}_{21} \mathrm{~N}_{3}$ & $\mathrm{C}_{14} \mathrm{~N}_{9}$ & $\mathrm{C}_{7} \mathrm{~N}_{15}$ & 470 & $\mathrm{C}_{31} \mathrm{~N}_{7}$ & $\mathrm{C}_{24} \mathrm{~N}_{13}$ & $\mathrm{C}_{17} \mathrm{~N}_{19}$ & $\mathrm{C}_{10} \mathrm{~N}_{25}$ \\
\hline 133 & $\mathrm{C}_{11} \mathrm{H}$ & $\mathrm{C}_{4} \mathrm{~N}_{6} \mathrm{H}$ & & 311 & $\mathrm{C}_{20} \mathrm{~N}_{5} \mathrm{H}$ & $\mathrm{C}_{13} \mathrm{~N}_{11} \mathrm{H}$ & $\mathrm{C}_{6} \mathrm{~N}_{17} \mathrm{H}$ & 478 & $\mathrm{C}_{34} \mathrm{~N}_{5}$ & $\mathrm{C}_{27} \mathrm{~N}_{11}$ & $\mathrm{C}_{20} \mathrm{~N}_{17}$ & $\mathrm{C}_{13} \mathrm{~N}_{23}$ \\
\hline \multirow[t]{2}{*}{139} & $\mathrm{C}_{8} \mathrm{~N}_{3} \mathrm{H}$ & & & 319 & $\mathrm{C}_{23} \mathrm{~N}_{3} \mathrm{H}$ & $\mathrm{C}_{16} \mathrm{~N}_{9} \mathrm{H}$ & $\mathrm{C}_{9} \mathrm{~N}_{15} \mathrm{H}$ & 495 & $\mathrm{C}_{33} \mathrm{~N}_{7} \mathrm{H}$ & $\mathrm{C}_{26} \mathrm{~N}_{13} \mathrm{H}$ & $\mathrm{C}_{19} \mathrm{~N}_{19} \mathrm{H}$ & $\mathrm{C}_{12} \mathrm{~N}_{25} \mathrm{H}$ \\
\hline & & & & 328 & $\mathrm{C}_{25} \mathrm{~N}_{2}$ & $\mathrm{C}_{18} \mathrm{~N}_{8}$ & $\mathrm{C}_{11} \mathrm{~N}_{14}$ & & & & & \\
\hline
\end{tabular}


Figure S7. Energies and structures of potential $\mathrm{C}_{4} \mathrm{~N}_{2} \mathrm{H}_{6}$ anion and neutral species with respect to the lowest energy anion. Structures were optimized at B3LYP and energies were calculated at $\operatorname{CCSD}(\mathrm{T}) / 6-311+\mathrm{g}(\mathrm{d})$. Ring structures were calculated to have negative electron affinities and vertical detachment energies; an example of the ring structure energies is shown with the asterisk.

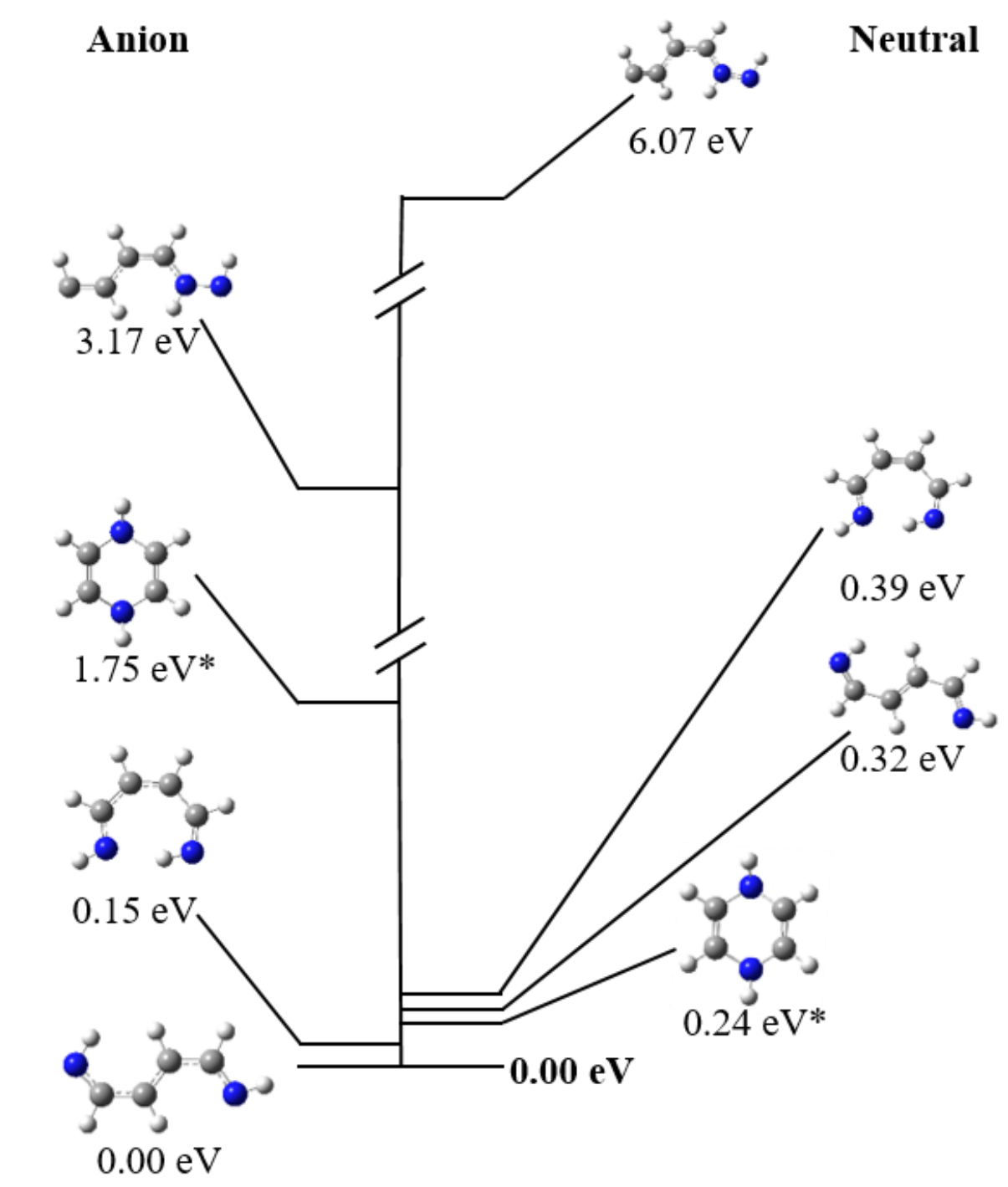


Figure S8. Energies and structures of potential $\mathrm{C}_{4} \mathrm{~N}_{2}$ anion and neutral species with respect to the lowest energy anion. Structures were optimized at B3LYP and energies were calculated at $\operatorname{CCSD}(\mathrm{T}) / 6-311+\mathrm{g}(\mathrm{d})$.

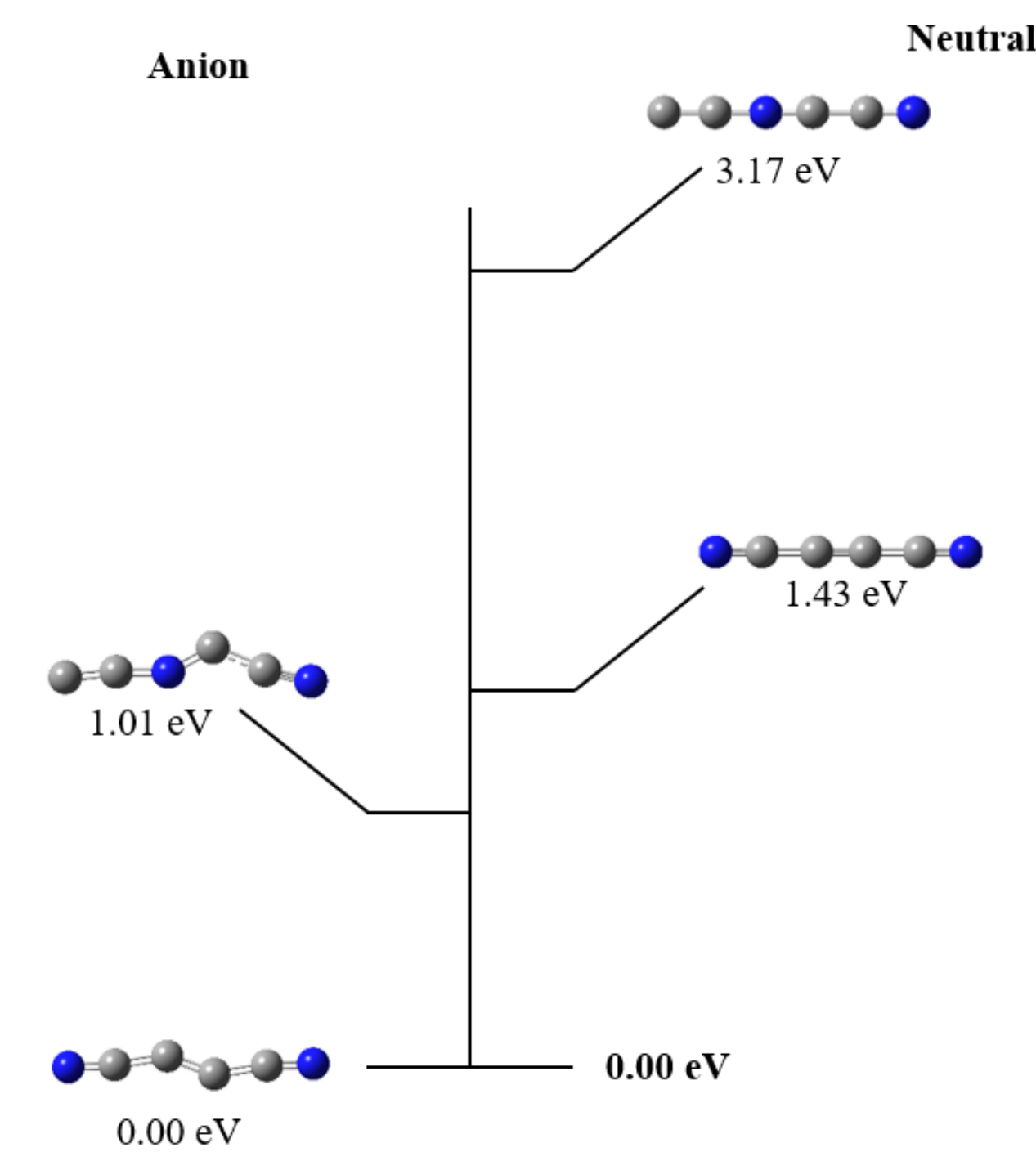


Table S12. Timing delays between the various triggers for laser ablation. For each value of $t$, several mass spectra were measured for incrementally increasing values of $t^{\prime}$. The values of $t^{\prime \prime}$ were varied to produce the cleanest mass spectrum in the low mass region. For $\mathrm{t}=100-200 \mu \mathrm{s}$, the cluster mass range was $0-132 \mathrm{~m} / \mathrm{z}$ (low mass region) and for $\mathrm{t}=300-400 \mu \mathrm{s}$, the cluster mass range was $60-1164 \mathrm{~m} / \mathrm{z}$ (high mass region).

\begin{tabular}{|c|c|c|c|}
\hline \multirow{t}{*}{$(\boldsymbol{\mu s})$} & $\mathbf{t}^{\prime}(\boldsymbol{\mu s})$ & $\mathbf{t}^{\prime \prime}(\boldsymbol{\mu} \mathbf{s})$ & Mass Range $(\boldsymbol{m} / \mathbf{z})$ \\
\hline \multirow{4}{*}{$\mathbf{1 0 0 , 1 5 0 , 2 0 0}$} & 210 & $1-2.5$ & $0-48$ \\
\cline { 2 - 4 } & 220 & $1.4-2.7$ & $0-96$ \\
\cline { 2 - 4 } & 230 & $1.6-3.8$ & $36-108$ \\
\cline { 2 - 4 } & 240 & $1.6-3.9$ & $48-132$ \\
\hline \multirow{3}{3}{$300,350,400$} & 320 & $3-4$ & $60-276$ \\
\cline { 2 - 4 } & 340 & 4 & $60-540$ \\
\cline { 2 - 4 } & 360 & $5-6$ & $84-996$ \\
\cline { 2 - 4 } & 380 & 6 & $84-1072$ \\
\cline { 2 - 4 } & 380 & 12 & $372-1164$ \\
\cline { 2 - 4 } & 400 & 7 & $144-1056$ \\
\hline
\end{tabular}


Powder X-ray diffraction patterns were collected on the PANalytical Empyrean instrument. X-rays were generated from a copper target with a wavelength $\mathrm{Cu} \mathrm{K}_{\alpha}$ of $1.5419 \AA$ A. Soller slits, anti-scatter slits, divergence slits, masks and a nickel filter were in the beam path. An X'Celerator linear strip detector, operating at $45 \mathrm{kV}$ and $40 \mathrm{~mA}$, was used in scanning mode with an active length of $2.1^{\circ}$. The scan ranges were $4^{\circ}-84^{\circ}$ for 1 and $4^{\circ}-85^{\circ}$ for 2 . All the diffraction patterns were collected using a step size of $0.017^{\circ}$. The counting time was 1600 s per increment for 1 and $1800 \mathrm{~s}$ for 2. During the measurement in reflection mode, the sample was spinning with a revolution time of $4 \mathrm{~s}$. The HighScore program (PANalytical) was used for processing XRD patterns including peak finding and fitting. The PXRD patterns for 1 and $\mathbf{2}$ are reported in Figure S9 and Figure S10. 
Figure S9. PXRD spectrum for $\mathbf{1}$

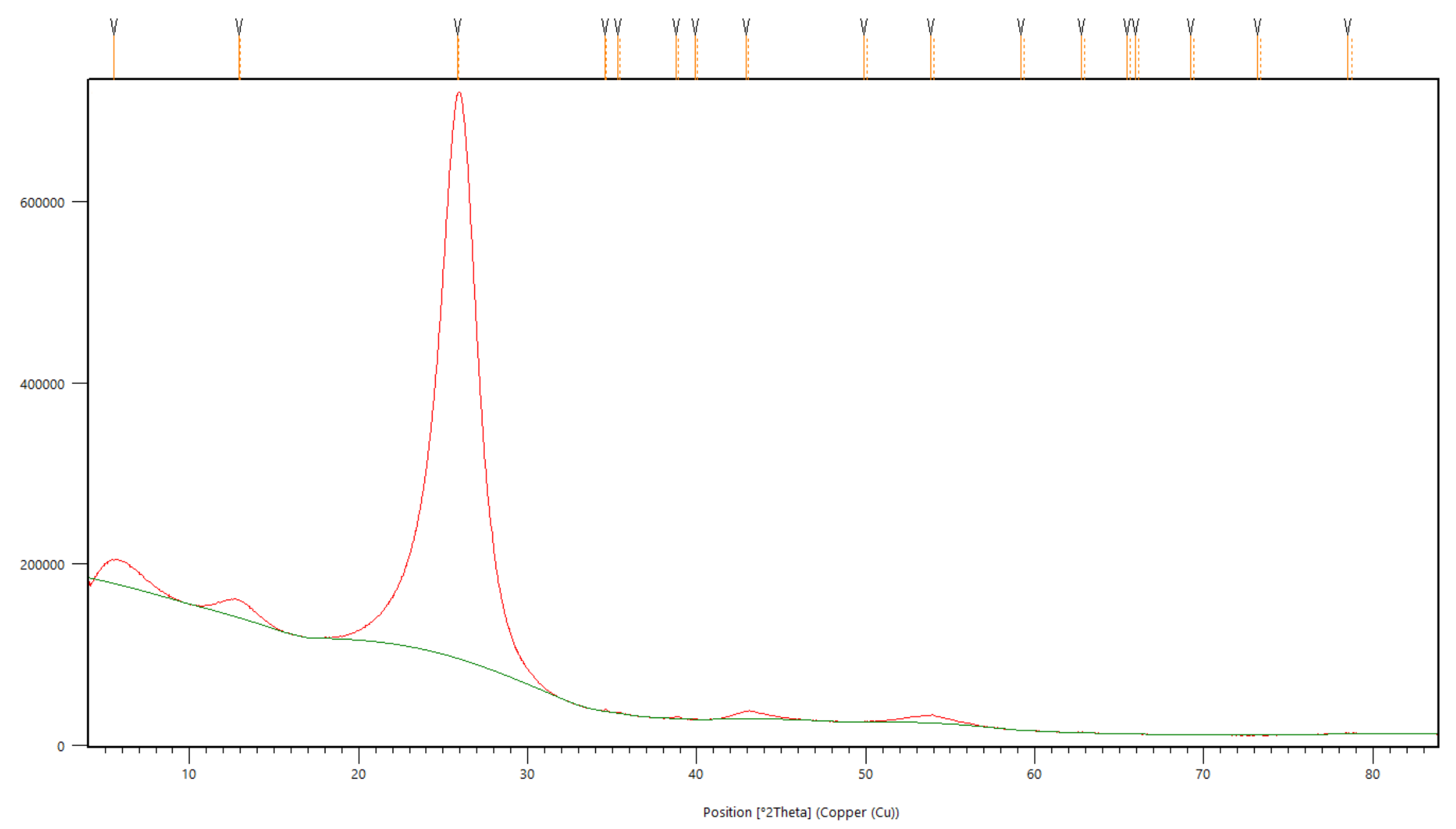


Figure S10. PXRD spectrum for 2

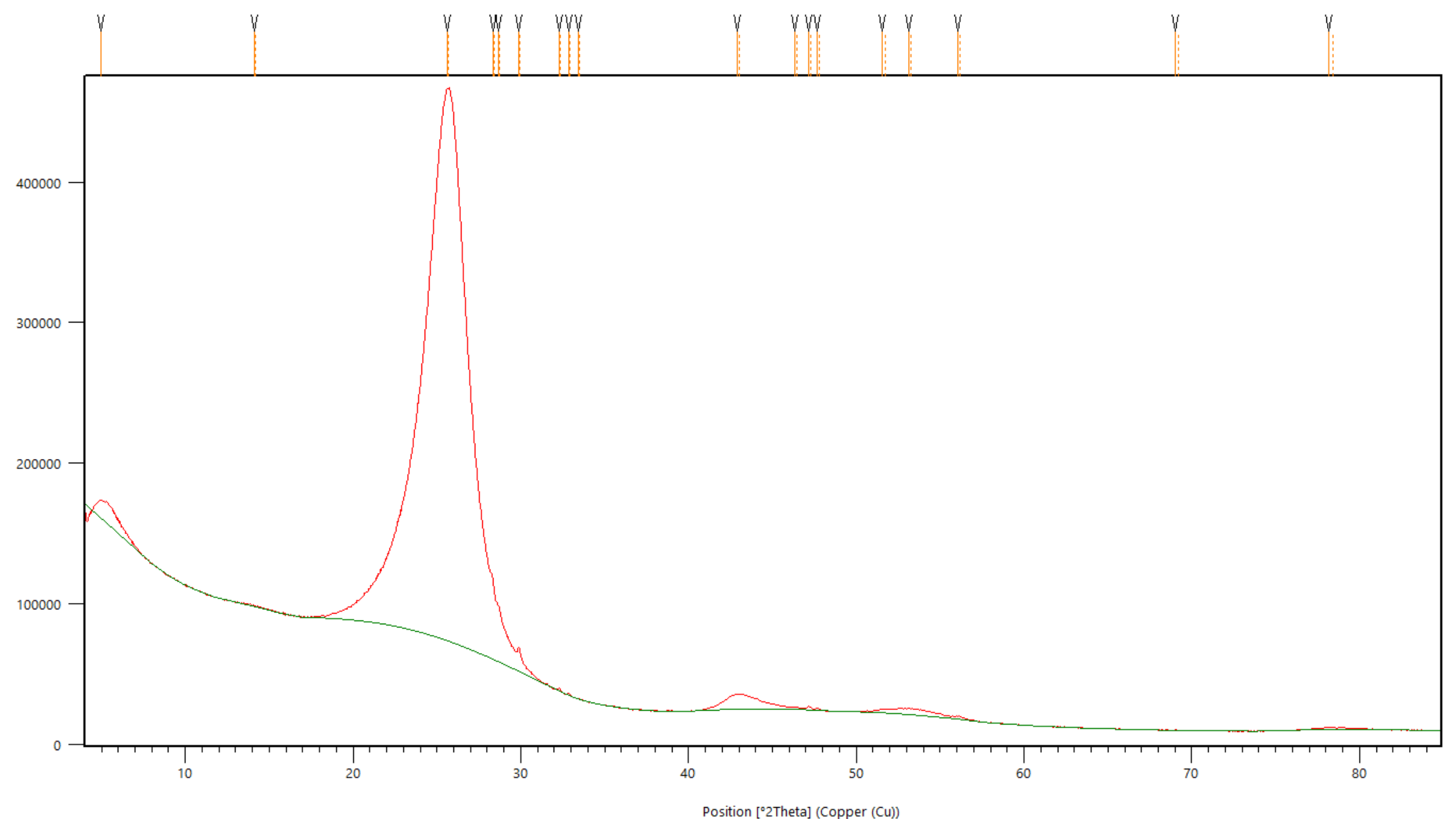

